\title{
Rheological Characterisation of Electrosterically Dispersed Alumina Suspensions During In Situ Coagulation
}

\author{
Jon G.P. Binner ${ }^{\star, \dagger, \ddagger}$ and Isabel Santacruz \\ Institute of Polymer Technology \& Materials Engineering, Loughborough University, Loughborough LE11 3TU, U.K. \\ Andrew M. McDermott ${ }^{\ddagger}$ \\ British Ceramic Tile, Newton Abbot, U.K.
}

\begin{abstract}
A near-net shape route for the production of advanced ceramic components has been developed that uses carboxylic acid derivatives as coagulants to achieve the destabilization of electrosterically dispersed high solids content ceramic suspensions via pH modification driving the suspension towards its IEP. In the present work, the effect of the time-dependent in situ hydrolysis of the coagulant, D-gulonic- $\gamma$-lactone, on the progressive destabilization of the precursor suspension to form highly viscous pastes has been characterized rheologically. The macroscopically determined dynamic storage and loss modulii, $G^{\prime}$ and $G^{\prime \prime}$, obtained during oscillatory investigations have been found to be sensitive rheological parameters that describe the structural changes occurring at a microscopic level within the material. The progression of coagulation has been found to be critically dependent on the coagulant concentration and suspension temperature. Increasing either of these variables greatly accelerates the extent and rate of coagulation. Furthermore, with judicious control over them, coagulation characteristics approaching idealized behavior can be obtained.
\end{abstract}

\section{Introduction}

I $\mathrm{N}$ many ways, current industrial ceramic forming technology has advanced little beyond the needs of traditional ceramics and consequently generally lacks measures to control microstructural homogeneity. This leads to the presence of small defects such as porosity, inclusions, and grain size variation throughout the structure. These both limit absolute component strength and cause large strength variations, often rendering components ineffective for high specification applications. In recent years, the importance of colloidal processing has been strongly emphasized as it offers the potential for significant control over the green microstructure. ${ }^{1}$ The approach involves dispersing ceramic powders of colloidal dimensions in a suitable medium, the resulting highly homogenous suspensions being consolidated either by removal of the suspending medium, as in slip casting, ${ }^{2}$ or by imparting a liquid-solid transition, for example, through gel or coagulation casting. Although slip casting is used extensively for traditional clay ware, its application to advanced ceramics is less common as it is a comparatively slow process.

There is, therefore, a need to produce dense, fine grained advanced ceramics using rheology-based near-net shape forming routes, with a reduction in the heterogeneities that are currently

R. E. Riman—contributing editor

Manuscript No. 20751. Received July 7, 2005; approved October 12, 2005.

Supported by Dytech Corporation Ltd., U.K. and the Engineering and Physical Science Research Council (EPSRC), U.K., via a CASE Studentship.

${ }^{*}$ Member, American Ceramic Society.

${ }^{\dagger}$ Author to whom correspondence should be addressed. e-mail: j.binner@lboro.ac.uk

${ }^{\ddagger}$ Author to whom correspondence should be addressed. e-mail: j.binner@lboro.ac.uk
${ }^{\ddagger}$ Formerly of the Department of Materials Engineering \& Materials Design, The University of Nottingham, U.K. present and on a faster production scale than is offered by slip casting. A wide variety of forming methods have been proposed recently, such as direct coagulation casting (DCC), ${ }^{3}$ gelcasting with acrylamides ${ }^{4}$ and polysaccharides, ${ }^{5,6}$ freeze casting, ${ }^{7}$ claylike forming/vibraforming, ${ }^{8,9}$ solid free-forming (SFF), ${ }^{10}$ temperature-induced forming (TIF), ${ }^{11-13}$ binder coagulation casting (BCC), ${ }^{14}$ etc. These approaches are capable of producing uniform, high-density bodies that contain fewer strength limiting defects than dry-processed components.

Graule, Gauckler, and co-workers ${ }^{3,15-20}$ have investigated and patented the DCC method for the production of complexshaped, high strength, high reliability ceramic components. The shape formation procedure was based on the destabilization of a high solids loading electrostatically stabilized suspension; destabilization being achieved by any one of the following methods ${ }^{19}$ : (a) changing the $\mathrm{pH}$ of the suspensions from a $\mathrm{pH}$ regime with high surface potential towards the IEP of the powder; (b) increasing the ionic strength in order to compress the electrostatic double layer of the powder particles; or (c) adsorbing charged ions on the powder surface in order to shift the IEP of the powder toward the actual $\mathrm{pH}$ of the suspension.

For the former approach, most of the work performed involved changing the $\mathrm{pH}$ from a basic $\mathrm{pH}$ towards a neutral or acidic $\mathrm{pH}$. Options included self-decomposing esters, enzymatic oxidation of sugars, e.g., glucose/glucosoxidase, ${ }^{19}$ or self-hydrolysable lactones such as gluconic acid lactone and glucuronic acid lactone. ${ }^{20-22}$ If a $\mathrm{pH}$ change from the acidic region toward a neutral $\mathrm{pH}$ was required then urea, formamide, acetamide, or hexamethylenetetramine (HMTA) could be used. High temperatures or the presence of determinate enzymes were required in order to achieve fast reactions. It was also shown to be possible to destabilize ceramic suspensions by increasing the salt concentration in the suspension by the internal formation ${ }^{19}$ or direct addition of salts. ${ }^{23}$

The strength of the wet-shaped, green body was found to be dependant on the coagulation method used. Flocculated bodies produced by a $\mathrm{pH}$ shift showed higher strengths than those coagulated by increasing the salt concentration as a result of the short-range hydration forces acting between the particles that occur in the latter case. ${ }^{17}$ The nature of the destabilization ion determined whether the body produced was flocculated or coagulated.

The current research also relies upon the in situ destabilization of a high solids loading suspension; however, it builds upon previous experience that focused on the coagulation of ceramic suspensions by the direct addition of salt to electrosterically stabilized suspensions. ${ }^{23}$ The latter process suffered from two inherent drawbacks. Firstly, mixing problems arose because the suspensions became highly viscous too quickly; this led to difficulties in obtaining bodies that were homogenous and free of air entrapment. Secondly, the scope of the process was limited to techniques that involved the subsequent plastic deformation of the pastes, e.g. extrusion. In order to alleviate these problems, the use of carboxylic acid derivatives, viz. lactones, as alternative 
coagulants was investigated. ${ }^{21,22}$ Lactones are cyclic carboxylic esters that interact with water in the high $\mathrm{pH}$ regime, opening their rings in order to form hydroxyl-carboxylic acids. Working at the same time as Gauckler and Graule, it was conceived that the time dependent in situ hydrolysis of this group of compounds would progressively destabilize suspensions that were electrosterically stabilized at basic $\mathrm{pH}$ via the formation of carboxylic acid, which would move the suspension toward its IEP. This would, in turn, yield the in situ coagulation of the suspension. The ideal viscosity-time behavior is shown in Fig. 1; the aim being to obtain a very rapid increase in viscosity. If this could be achieved while the suspension was contained within a non-porous mould then the final product would be a viscoelastic solid within which the initial homogeneity of the dispersion would be preserved. As a result, the components produced should possess high sintered densities and good mechanical properties.

\section{Experimental Procedure}

Commercial alumina (A-16SG, Alcoa Manufacturing (GB) Ltd., Worcester, U.K.) with a mean particle size of $0.5 \mu \mathrm{m}$ and a specific surface area of $9.2 \mathrm{~m}^{2} / \mathrm{g}$ was used as the ceramic powder. Aqueous suspensions were prepared to a solids loading of $81 \mathrm{wt} \%$ ( $51.8 \mathrm{vol} \%)$ by stirring the alumina powder into a premixed solution of dispersant and distilled water. An ammonium salt of carboxylic acid (Dispex A40, Allied Colloids, Bradford, U.K.), provided as a $40 \mathrm{wt} \%$ active content solution, was used as a deflocculant. Preparation of the initial dispersed suspensions followed the procedure developed by Davies and Binner, ${ }^{24}$ the dispersant being added at a level of $1.4 \times 10^{-3} \pm 0.001$ $\mathrm{g} / \mathrm{g}$ of alumina. After vigorous mechanical mixing, $300 \pm 0.01 \mathrm{~g}$ aliquots of the slurry were covered with Nescofilm (Nescofilm ${ }^{\mathrm{TM}}$, Nippon Shoji Kaisha Ltd., Osaka, Japan) to avoid contamination and left to stand at room temperature for $1 \mathrm{~h}$ to allow adsorption of the dispersant onto the alumina. In order to breakdown any soft agglomerates the suspension was exposed to ultrasonic energy (Model KS 150, Kerry Ultrasonics Ltd., Hitchin, U.K.) for $90 \mathrm{~s}$. The vibrational output was at a frequency of $20 \mathrm{kHz}$ and a power level of $150 \mathrm{~W}$. After that, the suspension was recovered with Nescofilm and cooled in a refrigerator at $7 \pm 2{ }^{\circ} \mathrm{C}$ for $1 \mathrm{~h}$. The dispersed suspensions were mechanically stirred and then placed in a $60 \pm 10 \mathrm{mmHg}$ vacuum for $1 \mathrm{~h}$ in order to remove as much of the entrapped air as possible. No significant evaporation was observed during this process. Finally, the suspensions were re-sealed and allowed to equilibrate in the refrigerator at $7 \pm 2{ }^{\circ} \mathrm{C}$ for $24 \mathrm{~h}$.

The slurry prepared in this way had a characteristic $\mathrm{pH}$ value (Jenway 3020 pH meter, Jencon Scientific Ltd., Leighton Buzzard, U.K.) of approximately $9.5 \pm 0.1$ at room temperature and

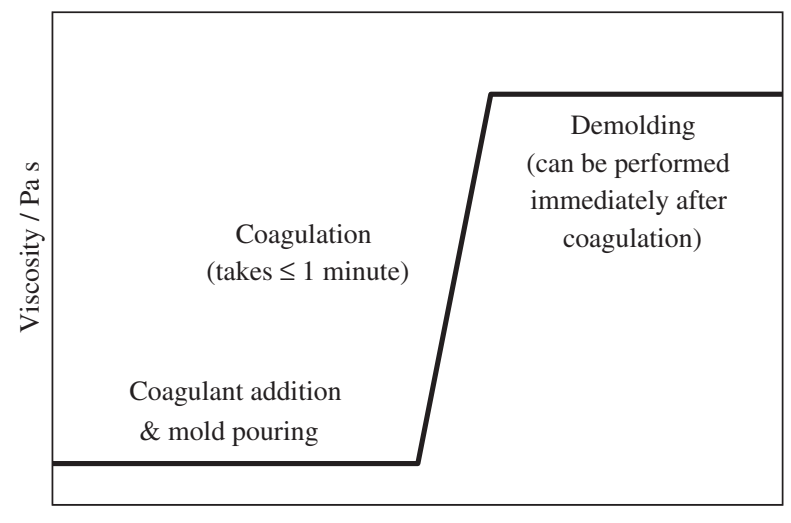

Time / $\min$

Fig. 1. Idealized time-dependent consistency increase for the in situ coagulation molding of ceramic suspensions. was easily pourable with an average viscosity of $0.5 \mathrm{~Pa} \mathrm{~s}$ at $109 \mathrm{~s}^{-1}$ shear rate.

Based on previous work that examined the consequence of lactone chemistry on suspension $\mathrm{pH}^{22}$ the effect of various concentrations of D-gulonic- $\gamma$-lactone (Aldrich, Gillingham, U.K.) on suspension rheology was investigated as a function of temperature. Additions of the coagulant are denoted by the number of moles of coagulant added per gram of unit mass of suspension, $\mathrm{mol} / \mathrm{g}$. For the in situ coagulation experiments the dispersed suspension was covered with Nescofilm to minimize evaporation and partially immersed in a gently agitated water bath to achieve constant temperature conditions. The cover was removed and the coagulant thoroughly blended into the suspension for $1 \mathrm{~min}$ prior to rheological measurements being made.

The suspensions were subjected to two forms of shear deformation, continuous and oscillatory, using two rotational instruments (Models V-88 and CS-10, both of them from Bohlin Instruments (U.K.), Cirencester, U.K.). The former is a constant strain viscometer capable of continuous shear flow characterization, while the latter is an advanced constant stress rheometer, capable of continuous shear and viscoelastic characterization. The latter also possessed a control loop allowing the stress to be regulated in order to provide operation in constant strain mode.

The experiments were designed to study the effect of the setting reactions on the deformation and flow properties of the suspensions such that inferences regarding interparticle potentials could be made. The effect of the lactone content between $1.00 \times 10^{-5}$ and $3.12 \times 10^{-5} \mathrm{~mol} / \mathrm{g}$, heating rate between $0.2^{\circ}$ and $3.0^{\circ} \mathrm{C} / \mathrm{min}$ and hold temperature between $7^{\circ}$ and $35^{\circ} \mathrm{C}$, were each studied during the in situ coagulation of suspensions. For the experiments focused on examining the effect of hold temperature, the suspensions were held initially at $15^{\circ} \mathrm{C}$ for $30 \mathrm{~min}$ to achieve equilibrium and then heated to different hold temperatures at a heating rate of $1^{\circ} \mathrm{C} / \mathrm{min}$.

\section{(1) Continuous Shear Deformation}

This technique was used to determine the viscosity of the coagulating sample under constant shear conditions. The V- 88 viscometer was used for these studies at a shear rate of $109 \mathrm{~s}^{-1}$ to match previous work. ${ }^{21-24}$ Two measurement geometries: coneplate (CP 5/30, cone diameter $30 \mathrm{~mm}$ and a cone angle of $5^{\circ}$ ) and parallel plate (PP 15, rotating upper plate $15 \mathrm{~mm}$ in diameter) were used depending on suspension viscosity, see Table I. Representative samples of the coagulating suspension were periodically removed from the resulting bulk slurry at known times after in situ coagulation had been initiated. After mounting on the viscometer the sample was immediately subjected to $60 \mathrm{~s}$ of preshear, prior to measurement, in order to reach equilibrium conditions. As the samples were prone to drying over extended periods, continuous evaluation was not possible and hence a fresh sample was utilized for each measurement.

\section{(2) Oscillatory Shear Deformation}

In order to study the viscoelasticity, small amplitude oscillatory rheometry was utilized using the CS-10 rheometer and parallel plate geometry (PP40, $40 \mathrm{~mm}$ in diameter), the latter was chosen because of its high-viscosity capability. The effect of time on the viscoelastic properties was investigated for samples subjected to a continuous oscillation at a frequency of $1 \mathrm{~Hz}$, the strain being

Table I. Viscosity Ranges Over which the Measuring Geometries were Operated at a Shear Rate of $109 s^{-1}$ on the V-88 Viscometer

\begin{tabular}{lc}
\hline Measuring configuration & Approximate viscosity range $(\mathrm{Pa} \cdot \mathrm{s})$ \\
\hline CP $5 / 30$ & $0.2-6$ \\
PP 15 & $2-16$ \\
\hline
\end{tabular}


kept constant at 0.01 by the autostress function. A maximum of five stress adjustments were used in order to regulate the strain at the desired amplitude and the acceptability factor was set at $\pm 5 \%$ of the target strain. In order to permit the rheology of the coagulating suspension to be measured continuously without the sample drying out, the solvent trap accessory was fitted around the mounted suspension and light paraffin oil (Paraffin Liquid, BDH Merck, Poole, U.K.) was used as a sealant. Temperature control was achieved through the use of the integral thermoregulated circulating water refrigeration/heating unit. The applied stress and the resulting oscillatory strain were automatically measured and analyzed to yield the complex shear modulus, $G^{*}$, dynamic storage modulus, $G^{\prime}$, dynamic loss modulus, $G^{\prime \prime}$, and phase angle, $\delta$. The viscoelastic data was collected every 30 or $60 \mathrm{~s}$ depending on the duration of the experiment as the software was inherently limited to a maximum number of 950 readings.

\section{Results}

\section{(1) Constant Shear Rheology}

The effect of lactone concentration and suspension temperature on the development of constant shear viscosity is shown in Figs. 2(a) and (b), respectively. In the former all the data relate to a coagulation temperature of $25^{\circ} \mathrm{C}$ while in the latter the lactone concentration was $2.52 \times 10^{-5} \mathrm{~mol} / \mathrm{g}$. The results show an initial

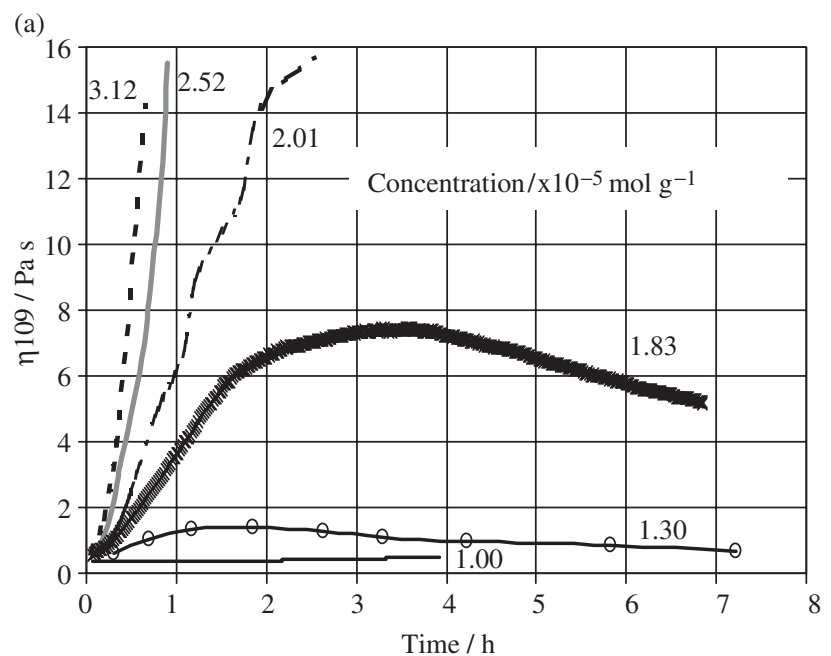

(b)

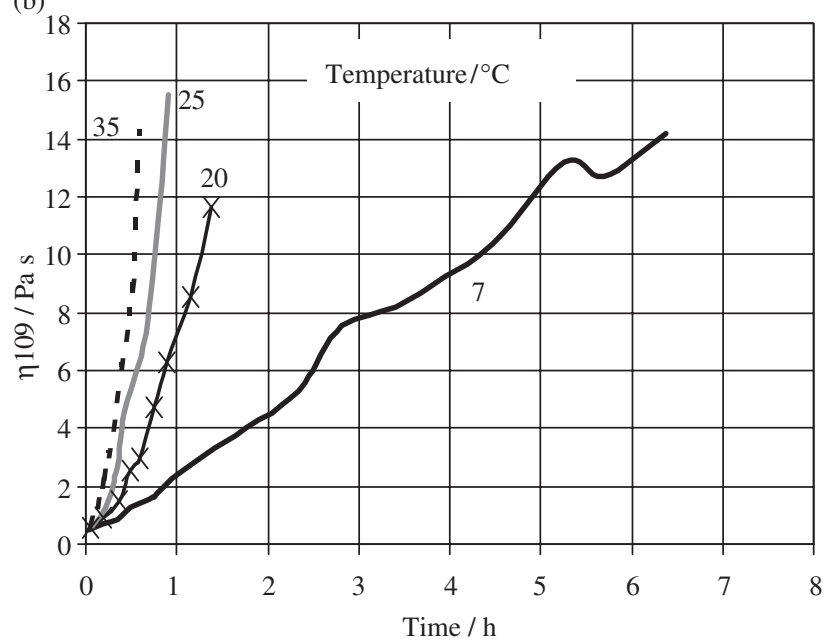

Fig. 2. Time-dependant change in constant shear suspension viscosity following the introduction of (a) various concentrations of D-gulonic- $\gamma$ lactone at $25^{\circ} \mathrm{C}$ and (b) $2.52 \times 10^{-5} \mathrm{~mol} / \mathrm{g}$ of lactone as a function of temperature. increase in suspension viscosity with time. However at extended periods, a partial reduction in viscosity is noted, at least at low coagulant levels, prior to establishing an equilibrium value. As was observed for the effect of these parameters on $\mathrm{pH}$ change, reported elsewhere, ${ }^{22}$ both larger quantities of lactone and higher temperatures favor a more rapid initial rise with higher peak and equilibrium viscosities.

\section{(2) Oscillatory Shear Rheology}

The effect of the addition of $2.50 \times 10^{-5} \mathrm{~mol} / \mathrm{g}$ of lactone on the dispersed suspension at $25^{\circ} \mathrm{C}$ is depicted in Fig. 3. The notable feature is that $G^{*}, G^{\prime}$, and $G^{\prime \prime}$ all increased throughout the duration of the experiment. Analysis of $G^{\prime}$ (elastic response) and $G^{\prime \prime}$ (viscous response) illustrated a particularly significant trend in that the relative increments were not the same; $G^{\prime}$ was initially lower than $G^{\prime \prime}$, however as coagulation proceeds, $G^{\prime}$ surpassed $G^{\prime \prime}$. When testing within the linear region, not all dynamic properties need to be determined because they are all interrelated. In this study $G^{\prime}$ and $\delta$ were the most informative parameters as they clearly indicated the development of elasticity, or solid-like behavior, in the body as in situ coagulation proceeded. Higher values of $G^{\prime}$ denote greater solid-like behavior, while $\delta$ takes values of $0^{\circ}$ for ideal solids and $90^{\circ}$ for ideal liquids.

The effect of lactone concentration and suspension temperature on the time-dependent evolution of $G^{\prime}$ and $\delta$ are illustrated in Figs. 4 and 5. Once again, the data in the former relates to a coagulation temperature of $25^{\circ} \mathrm{C}$, while the lactone content in the latter was $1.99 \times 10^{-5} \mathrm{~mol} / \mathrm{g}$. Upon increasing either the lactone concentration or temperature, $G^{\prime}$ evolved faster and attained a higher peak value, although at extended times a decrease was apparent. A similar trend was observed for $\delta$. A summary of the effect of lactone concentration on the time required to attain coagulation, the gelation time, $t_{\mathrm{gel}}$, is depicted in Fig. 6. The latter can be defined as the point where the elastic modulus, $G^{\prime}$, displays the same value as the viscous modulus, $G^{\prime \prime}$, hence the angle, $\delta$ is $45^{\circ}$. This is of rheological significance because at this point the material possesses $50 \%$ elastic and $50 \%$ viscous behavior. This experimental protocol is in accordance with the ASTM D 4473 standard.

Another experimental factor that influences the in situ coagulation of a suspension is the temperature profile to which it is exposed, i.e., the hold temperature and heating rate. The effect of these is illustrated in Figs. 7(a) and (b), respectively; it can be seen that they have a marked effect on the evolution of $G^{\prime}$. Note that in these figures, the $30 \mathrm{~min}$ equilibration time at $15^{\circ} \mathrm{C}$ has been included in the time plotted on the $x$-axis.

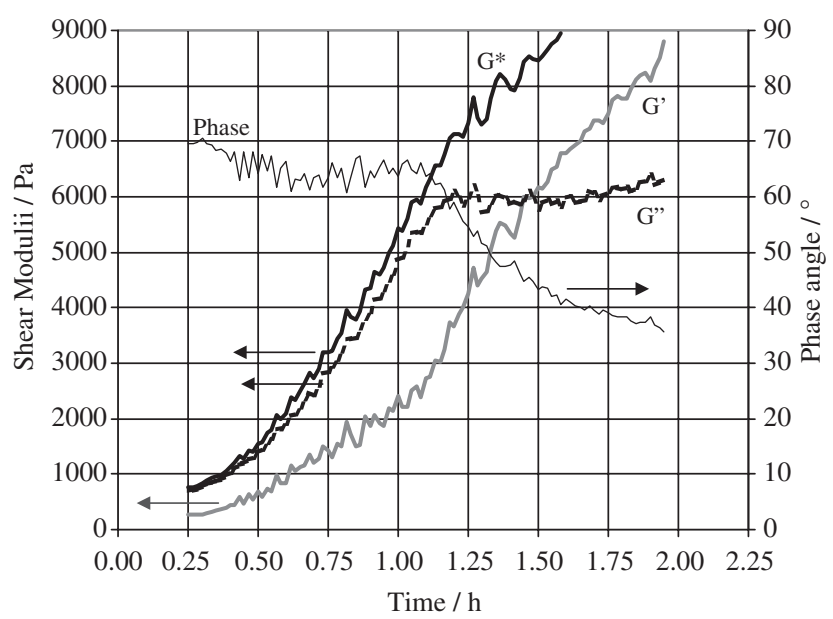

Fig. 3. Dynamic oscillatory data as a function of time for the coagulation of a standard dispersed suspension using $2.5 \times 10^{-5} \mathrm{~mol} / \mathrm{g}$ of $\mathrm{D}^{-}$ gulonic- $\gamma$-lactone at $25^{\circ} \mathrm{C}$. 
(a)
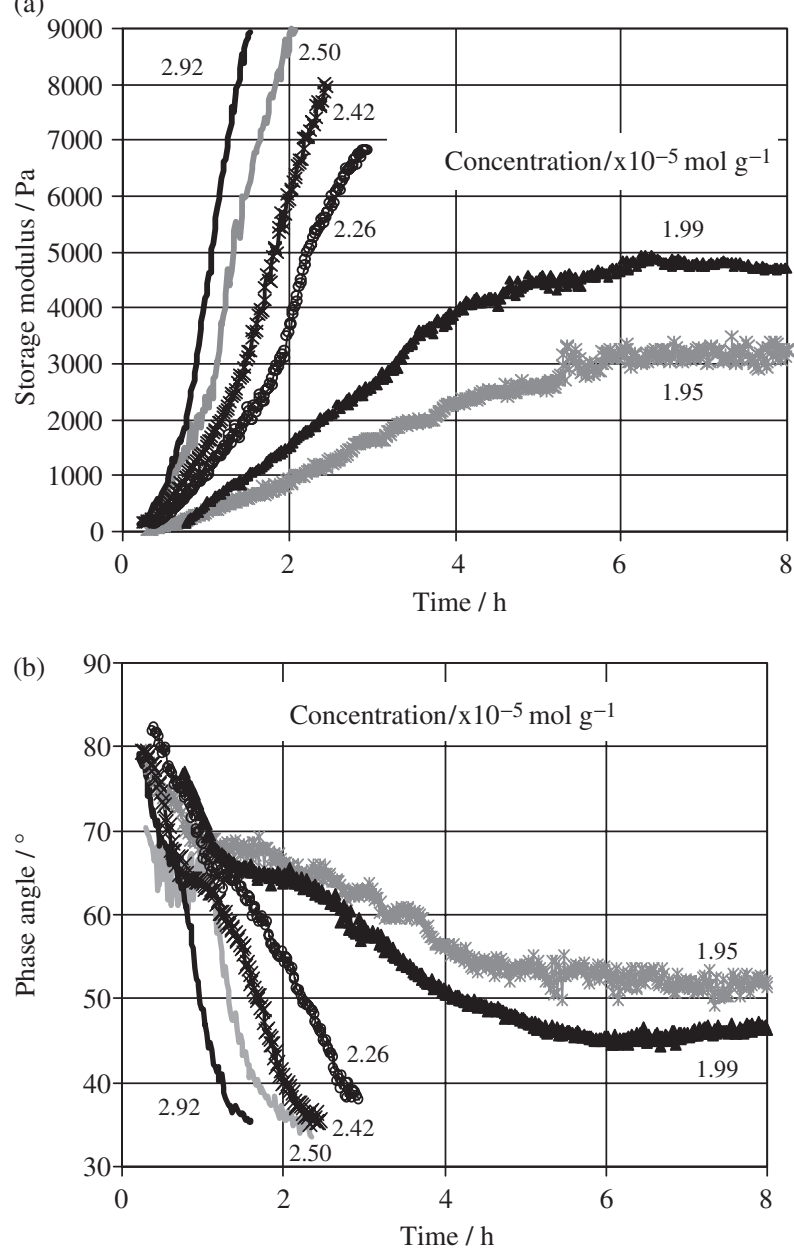

Fig. 4. Concentration dependence at $25^{\circ} \mathrm{C}$ for (a) storage modulus and (b) phase angle for additions of D-gulonic- $\gamma$-lactone.

\section{Discussion}

Figure 1 depicts a model system in which an ideal time-dependant viscosity increase is shown for the in situ coagulation molding route following the addition of coagulant. The model is composed of three distinct stages: (1) coagulant addition and mold pouring; (2) in situ coagulation; and (3) demolding.

The addition of the coagulant to the suspension, and subsequent pouring into the mold, during the first stage of the process should result in a negligible increase in viscosity relative to the precursor suspension, this will allow thorough homogenization of the coagulant into the suspension, the complete removal of entrapped air and the complete filling of even complex-shaped molds. Ideally, a time of no more than 5-10 min should be sufficient for all of these stages though it would also be useful if the suspension could be held for long periods (e.g., over weekends) with the coagulant already added. It is worth noting that above a viscosity of $\eta_{109} \approx 2 \mathrm{~Pa}$ s or $G^{\prime} \approx 300 \mathrm{~Pa}$ the suspension becomes too viscous to permit adequate de-airing and pouring. As soon as the suspension has been introduced into the mold cavity, it is desirable to trigger a very rapid increase in consistency. As far as can be determined from the present work, the coagulation process itself cannot happen too quickly, i.e., there are no negative consequences from having coagulation occur almost instantaneously, provided the first stage has been performed successfully. Finally, during demolding a steady-state value for viscosity would be beneficial, allowing demolding to occur at a time convenient to the operator. Throughout this period the body must be sufficiently stiff to prevent any deformation during demolding or subsequent handling.

The rheological measurements described below were performed on the suspensions during in situ coagulation in order
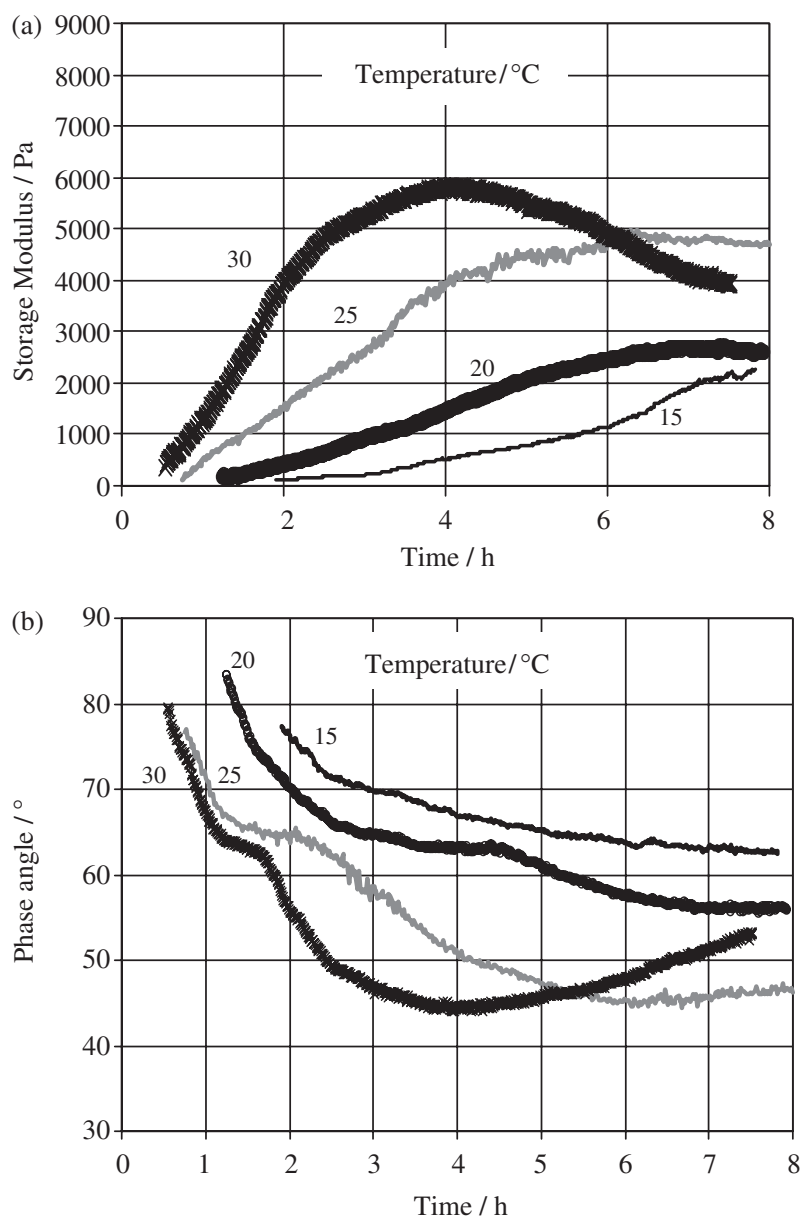

Fig. 5. Temperature dependence for (a) storage modulus and (b) phase angle for $2.00 \times 10^{-5} \mathrm{~mol} / \mathrm{g}$ additions of D-gulonic- $\gamma$-lactone.

to follow their destabilization as the $\mathrm{pH}$ approached the suspension IEP, from basic towards the neutral-acidic region, because of the hydrolysis of the lactone.

\section{(1) Constant Shear Rheology}

The constant shear rate measurements offer relevant information about the evolution of the suspension during in situ coagulation and can give some idea about the time required before demolding as the latter is related to the viscosity of the paste formed. Based on previous work, ${ }^{22,23}$ a shear rate of $109 \mathrm{~s}^{-1}$ was selected for all these measurements as the structure formed during the coagulation is sensitive to high shear. The results ob-

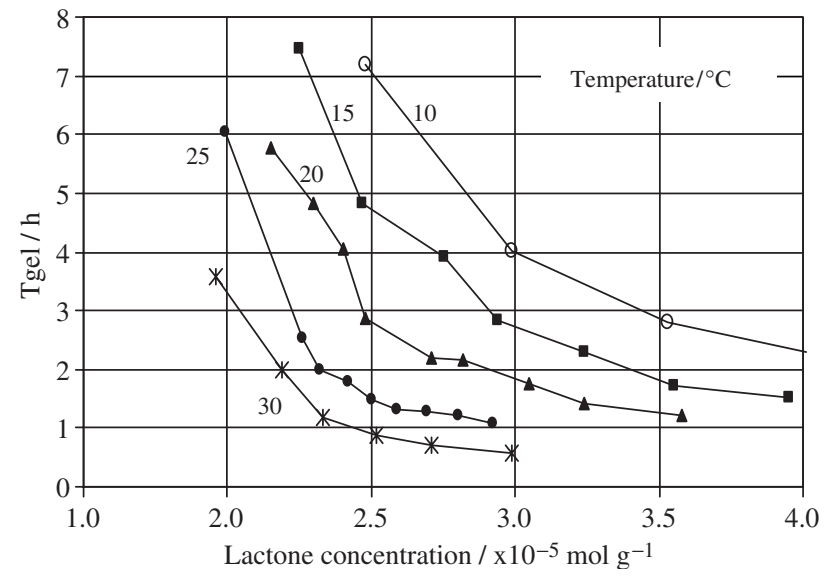

Fig. 6. Variation in time required for the suspension to attain $t_{\text {gel }}$ as a function of lactone concentration and temperature. 

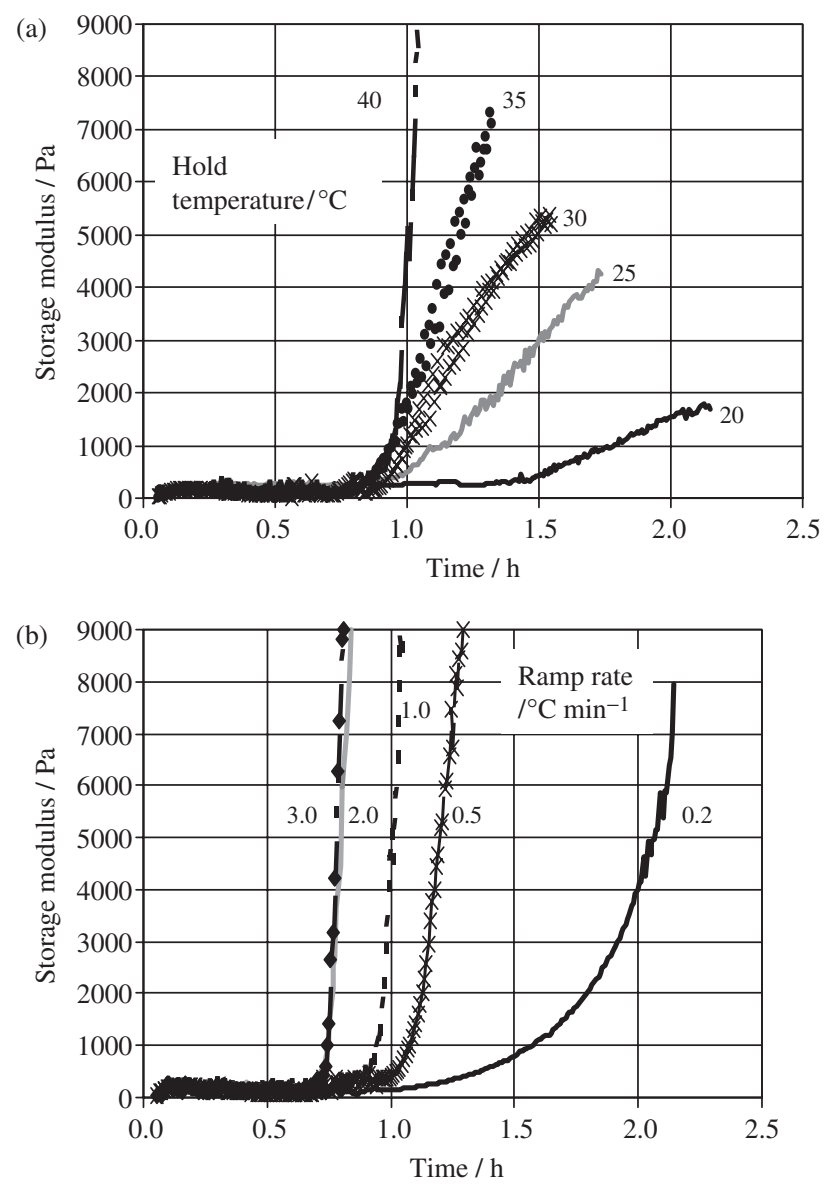

Fig. 7. Effect of (a) hold temperature and (b) heating rate on the evolution of $G^{\prime}$ during suspension coagulation.

tained were complementary to those from the oscillatory measurements.

The initial rise in viscosity in Fig. 2 is attributable to the progressive destabilization of the initial dispersion by the in situ formation of acid as a result of the hydrolysis of the lactone to yield $\mathrm{H}^{+}$ions that neutralize the negative charges associated with the polyelectrolyte, reducing its contribution to repulsion. The decrease in $\mathrm{pH}$ induces a conformational change on the polyelectrolyte from the extended tail conformation to a more dense and compact loop conformation. The polyelectrolyte chains become uncharged and the formation of loops in the adsorbed configurations is enhanced. Thus, the projected surface area per adsorbed chain is relatively small and more adsorbed chains are required to establish a saturated monolayer. ${ }^{25}$ Hence the decrease in the $\mathrm{pH}$ is believed to reduce both the steric component of the initial electrosteric repulsion as a result of the change in polymer conformation and also the electrostatic component, the latter due both to the new conformation of the polymer and to the ions around the particle forming the double layer, which is reduced close to the IEP.

While the resulting level of repulsion is unable to continue to provide dispersion, the thin steric barrier associated with the dispersant is effective at preventing the particles from touching. This permits particle rearrangement and hence high packing density bodies are produced. It can be seen from the figure that a larger concentration of lactone favors both the extent and rate of in situ coagulation. These observations are in direct accordance with the thermodynamic and kinetic considerations discussed elsewhere. ${ }^{22}$

Interestingly, the viscosity of the low lactone content suspensions decreased again at extended times after the in situ coagulation process had occurred (Fig. 2). It might also have occurred for the high lactone content suspensions but was not observed.
There are two possible explanations for this behavior. Firstly, a further conformational change for the polyelectrolyte from the compact loop form to a more extensive equilibrium arrangement $^{24}$ led to a decrease in rigidity. Secondly, the increase in ionic strength that occurs as well as the conformation change only increases the viscosity for a short time because the shrinking double layer leads to lower effective solids content. ${ }^{26}$ The consequence of this behavior is that the time available before demolding is limited, although as it is several hours this should not cause any practical problems with the process.

\section{(2) Oscillatory Shear Rheology}

It was shown in Fig. 3 that as coagulation proceeds, both $G^{\prime}$ and $G^{\prime \prime}$ increased with time and eventually $G^{\prime}$ could become greater than $G^{\prime \prime}$, i.e., although some elasticity was present in the initial ceramic suspension because of its viscoelastic behavior, its relative importance increased during in situ coagulation. This type of behavior, whereby the solid-like properties progressively develop at a faster rate than the viscous properties, is commonly observed in systems undergoing rigidification. ${ }^{27}$ At extended times, the high magnitude of the ratio $G^{\prime} / G^{\prime \prime}$ indicated that a stiff viscoelastic solid had been formed.

The observed changes in viscoelasticity can be explained by considering the destabilization process that occurs during in situ coagulation. During the initial stages of coagulation the repulsive interparticle potential dominates, resulting in a low $G^{\prime}$. However as the process proceeds the interparticle repulsion progressively becomes reduced such that the importance of the attractive interaction increases. This permits closer particle approach and leads to the development of structure within the material. As a result, it is convenient to consider the storage modulus as a measure of the extent of interparticle attraction.

In Figs. 4 and 5 it can be seen that, as expected, faster coagulation is observed at higher concentrations of lactone and at higher temperatures, because of the faster kinetics in reducing the suspension $\mathrm{pH}$. As observed during the constant shear viscosity measurements, the suspensions with low lactone content also display a maximum in their solid-like behavior prior to partial relaxation. This effect was discussed above.

At the gel point, $t_{\mathrm{gel}}$, which represents the crossover point between the dynamic modulii at this frequency of measurement, the system ceased to be dominated by liquid-like properties and began to behave as a solid, so much so that it was found that the body could be demolded once this point had been reached. Figure 6 shows that as either the concentration of lactone added to the system or the suspension temperature increased, the time taken to attain the $t_{\text {gel }}$ decreased. However, close examination of the underlying data behind Figs. 3-5 revealed that the magnitude of the crossover point was independent of the lactone concentration or temperature used and, for the selected rheological parameters of a $1 \mathrm{~Hz}$ measurement frequency and a strain of 0.01 , always occurred at $\sim 6000 \mathrm{~Pa}$. Durand et al. ${ }^{28}$ investigated the gelation of pectin and also found a similar independence. This made the gel point a useful tool in aiding the assessment of optimal processing conditions.

The general trends associated with the lactone concentration and the temperature are usefully indicated by superimposing both sets of data to form an "iso-gel point time" contour profile (Fig. 8). This plot was valuable in identifying the time required for the suspension to reach the gel point, i.e., the demolding time, for any combination of concentration and temperature used. However, as shown by Fig. 7, the application of variable temperature profiles is an additional processing parameter that can be exploited usefully to control the setting characteristics and achieve coagulation profiles. For example, maintaining the suspension temperature at $15^{\circ} \mathrm{C}$ after coagulant addition resulted in a minimal initial increase such that thorough homogenization and de-airing were possible. Even after $30 \mathrm{~min}$ at this temperature the suspension was still fluid enough to be poured. Subsequently, raising the temperature at $3^{\circ} \mathrm{C} / \mathrm{min}$ to $40^{\circ} \mathrm{C}$ resulted in rapid coagulation, allowing demolding after only 5-10 


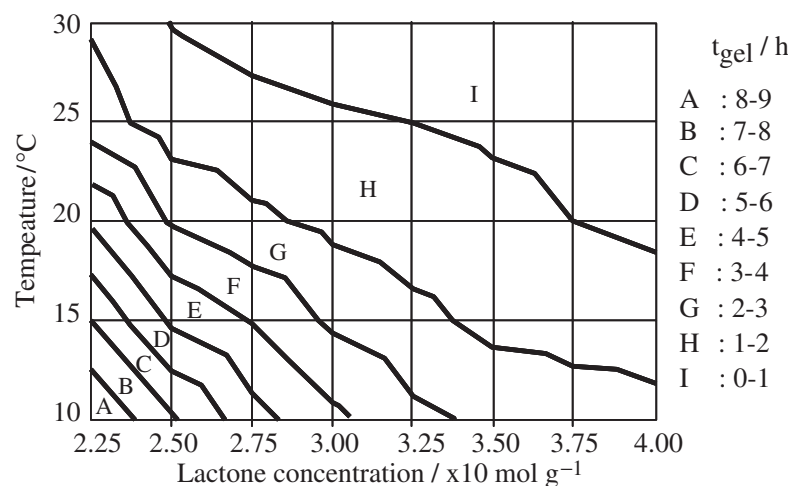

Fig. 8. Contour plot indicating the time required to reach $t_{\text {gel, }}$ as a function of lactone concentration and temperature. The lines represent times of equal magnitude.

min. Faster heating rates, and hence shorter times before demolding, may be achievable, for example by using microwaves, but were not investigated in the present work.

\section{Conclusions}

It has been demonstrated that dispersed suspensions can be successfully coagulated in a time-dependent manner using D-gulonic- $\gamma$-lactone. This chemical is initially inactive but hydrolyses over a period of time to produce a carboxylic acid. The presence of this acidic component subsequently causes the suspension to coagulate. As a result of the initially passive nature of this additive, it can be thoroughly mixed into the dispersion such that coagulation is achieved entirely homogeneously. The progression of in situ coagulation is critically dependent on the coagulant concentration and suspension temperature. Increasing either of these variables greatly accelerates the extent and rate of coagulation.

The changes in suspension properties as they become highly viscous pastes have been characterized rheologically. The macroscopically determined modulii, $G^{\prime}$ and $G^{\prime \prime}$, obtained during oscillatory investigations are sensitive rheological parameters that describe the structural changes occurring at a microscopic level within the material. The in situ coagulation process results in the formation of a solid-like network that exhibits strongly elastic behavior from an initial viscous-dominated fluid. Furthermore, the ability of this technique to monitor these changes over a much wider consistency range is of considerable advantage.

While this coagulation casting technique is never going to be able to compete with dry forming routes such as die or isostatic pressing in terms of shear speed, it does appear to be able to offer the ability to produce complex-shaped ceramics with homogeneous microstructures at a faster rate than other colloidalbased techniques, such as slip casting.

\section{References}

${ }^{1}$ F. F. Lange, "Powder Processing Science and Technology for Increased Reliability," J. Am. Ceram. Soc., 72 [1] 3-15 (1989).
${ }^{2}$ R. E. Cowan, "Treatise on Materials Science and Technology"; pp. 153-71 in Ceramic Fabrication Processes, Vol. 9, Edited by F. F. Y. Wang. Academic Press, New York, 1976.

${ }^{3}$ B. Balzer, M. K. M. Hruschka, and L. J. Gauckler, "Coagulation Kinetics and Mechanical Behavior of Wet Alumina Green Bodies Produced via DCC," J. Colloid Interface Sci., 216, 379-86 (1999).

${ }^{4}$ O. O. Omatate, "Gelcasting - A New Ceramic Forming Process," Am. Ceram. Soc. Bull., 70 [10] 1641-9 (1991).

${ }^{5}$ A. J. Fanelli, R. D. Silvers, W. S. Frei, J. V. Burlew, and G. B. Marsh, "New Aqueous Injection Molding Process for Ceramic Powders," J. Am. Ceram. Soc., 72 [10] 1833-6 (1989).

${ }^{6}$ I. Santacruz, M. I. Nieto, and R. Moreno, "Rheological Characterization of Synergistic Mixtures of Carrageenan and Locust Bean Gum for Aqueous Gelcasting of Alumina," J. Am. Ceram. Soc., 85 [10] 2432-6 (2002).

${ }^{7}$ S. W. Sofie and F. Dogan, "Freeze Casting of Aqueous Alumina Slurries with Glycerol," J. Am. Ceram. Soc., 84 [7] 1459-64 (2001).

${ }^{8}$ B. V. Velamakanni, J. C. Chang, F. F. Lange, and D. S. Pearson, "New Method for Efficient Colloidal Particle Packing via Modulation of Repulsive Lubricating Hydration Forces," Langmuir, 6, 1323-5 (1990).

${ }^{9}$ G. V. Franks and F. F. Lange, "Vibraforming and In-Situ Flocculation of Consolidated, Coagulated, Alumina Slurries," J. Am. Ceram. Soc., 78 [5] 1324-8 (1995).

${ }^{10}$ S. Baskaran, G. D. D. Maupin, and G. L. Graff, "Free-form Fabrication of Ceramics," Am. Ceram. Soc. Bull., 76 [7] 53-8 (1998).

${ }^{11}$ N. S. Bell, L. Wang, W. M. Sigmund, and F. Aldinger, "Temperature Induced Forming: Application of Bridging Flocculation to Near-Net Shape Production of Ceramic Parts," Z. Metallkd, 90 [6] 388-92 (1999).

${ }^{12}$ L. Wang, W. Sigmund, and F. Aldinger, "Parameters that Influence the Temperature Induced Forming"; pp. 477-82 in Ceramic Transactions, Vol. 112, Ceramic Processing Science VI, Edited by S. Hirano, G. L. Messing, and M. Clausen. American Ceramic Society, Westerville, OH, 2001.

${ }^{13}$ E. Ewais, A. A. Zaman, and W. Sigmund, "Temperature Induced Forming of Zirconia from Aqueous Slurries: Mechanism and Rheology," J. Eur. Ceram. Soc., 22, 2805-12 (2002).

${ }^{14}$ W. J. Jr. Walker, D. K. Chatterjee, S. K. Ghosh, and J. S. Reed, "Binder Coagulation Casting of Ferroelectric Components," Ferroelectrics, 231, 279-84 (1999).

${ }^{15}$ T. J. Graule and L. J. Gauckler. "Process for Producing Ceramic Green Bodies," EU Patent EP0605694V (1994) and US Patent US5667548 (1997).

${ }^{16}$ T. J. Graule and L. J. Gauckler, "Shaping of Ceramic Green Components Direct from Suspensions by Enzyme Catalysed Reactions," Deutsche Keramische Gesellschaft, 71 [6] 317-23 (1994).

${ }^{17}$ F. H. Baader, T. J. Graule, and L. J. Gauckler, "Direct Coagulation CastingA New Green Shaping Technique. Part I: Application to Alumina," Ind. Ceram., 16 [1] 31-6 (1996).

${ }^{18}$ F. H. Baader, T. J. Graule, and L. J. Gauckler, "Direct Coagulation CastingA New Green Shaping Technique. Part II: Application to Alumina," Ind. Ceram., 16 [1] 36-40 (1996).

${ }^{19}$ L. J. Gauckler, T. J. Graule, and F. Baader, "Ceramic Forming Using Enzyme Catalyzed Reactions," Mater. Chem. Phys., 61, 78-102 (1999).

${ }^{20}$ A. R. Studart, V. C. Pandolfelli, E. Tervoort, and L. J. Gauckler, "In Situ Coagulation of High-Alumina Zero-Cement Refractory Castables," J. Am. Ceram. Soc., 85 [8] 1947-53 (2002).

${ }^{21}$ J. G. P. Binner, A. M. McDermott, Y. Yin, R. M. Sambrook, and B. Vaidhyanathan, "In-Situ Coagulation Moulding: A New Route for High Quality, Net Shape Ceramics," Ceram. Int. 32 [1] 29-35 (2006).

${ }^{22}$ Y. Yin, J. G. P. Binner, M. J. Hey, and J. R. Mitchell, "Hydrolysis of Carboxylic Lactones in Alumina Slurries," J. Eur. Ceram. Soc. in press.

${ }^{23}$ J. Davies and J. G. P. Binner, "Coagulation of Electrosterically Dispersed Concentrated Alumina Suspensions for Paste Production," J. Eur. Ceram. Soc., 20, 1555-67 (2000).

${ }^{24}$ J. Davies and J. G. P. Binner, "The Role of Ammonium Polyacrylate in Dispersing High Solids Content Alumina Suspensions," J. Eur. Ceram. Soc., 20, 1539-53 (2000).

${ }^{25}$ J. Cesarano III, I. A. Aksay, and A. Bleier, "Stability of Aqueous $\alpha-\mathrm{Al}_{2} \mathrm{O}_{3}$ Suspensions with Poly (Methacrylic Acid) Polyelectrolyte," J. Am. Ceram. Soc., 71 [4] 250-5 (1988).

${ }^{26}$ B. Balzer, M. K. M. Hruschka, and L. J. Gauckler, "In Situ Rheological Investigations of the Coagulation in Aqueous Alumina Suspensions," J. Am. Ceram. Soc., 84 [8] 1733-9 (2001).

${ }^{27}$ N. Kiratzis and P. F. Luckham, "Rheological Behaviour of Stabilised Aqueous Alumina Dispersions in the Presence of Hydroxyethyl Cellulose," J. Eur. Ceram. Soc., 18, 783-90 (1998).

${ }^{28}$ D. Durand, C. Bertrand, H. Clark, and A. Lips, "Calcium-Induced Gelation of Low Methoxy Pectin Solutions-Thermodynamic and Rheological Considerations," Int. J. Biol. Macromolec., 12 [1] 14-18 (1990). 